\title{
Virtual Observatory as a tool for stellar spectroscopy
}

\author{
Petr Škoda \\ Astronomical Institute of the Academy of Sciences of the Czech Republic, \\ Fričova 298, 25165 Ondřejov \\ email: skoda@sunstel.asu.cas.cz
}

The Virtual Observatory (VO) is often presented as an all-purpose tool for the astronomer to do all his current work in a single web browser, from which he can control all data searches, reductions and mainly the full data analysis. This idea seems to be already fulfilling in certain fields of astronomy like is the search of unknown correlations between various parameters in large scale surveys, galaxy statistics or cross-identification of objects. The current VO tools are, however, lacking support for important capabilities needed in stellar spectroscopy. Especially the high-resolution spectroscopy of stars with rapid line profile variations or of those with complicated emission profiles (like Be or symbiotic stars) benefits from a number of specific methods implemented today only in stand-alone legacy packages working with local data.

We concentrate on such methods and give recommendations for their implementation as a VO services using both remote and local spectra in an unified manner as well as the distributed computing power or nice graphics available in current VO infrastructure.

Among the common tasks required for analysis of stellar spectra belong mainly: overplotting many spectra of the same object in time or folded by phase, measurement of radial velocities or red-shift (using fitting of profiles by various functions, profile mirroring around given laboratory wavelength or cross-correlation with reasonable templates), bisector and moment analysis of line profiles, complex period analysis (mostly using PDM or CLEAN methods), dynamic spectra (quotient or differential, showing line profile changes in time or in dependence on orbital phase) and for multiple stars there is a very powerful method of spectra disentangling (in wavelength domain or in Fourier space).

Implementing these tasks as VO services would benefit from the already existing VO infrastructure like is unified data format (VO-Table, UCD semantics of variables), transparent data conversion (including the physical unit transformation already available in SSAP), on-the-fly rescaling as well as powerful display and presentation tools capable of seamless work with remote data.

The precise analysis of optical stellar spectra requires in addition the introduction of certain capabilities into the current VO-enabled spectral tools. For example the flexible curve fitting using more complicated functions than simple polynomials or splines (e.g., INTEP or AKIMA methods) is necessary for good normalisation of the continuum or the measurement of radial velocities sometimes needs different fits for the core and line wings.

Therefore, we also give a review of several VO-compatible spectral tools (SpecView, SPLAT and VOSpec) emphasising their deficiencies and advantages for these tasks.

We hope our review will help the VO developers to identify the most important features to concentrate on in further improvements of VO-enabled spectral tools to allow the fullfledged analysis of stellar spectra to be done in VO domain. 